\title{
TOPOLOGICAL 3D ELEVATION DATA INTERPOLATION OF ASTER GDEM BASED ON CONTINUOUS DEFORMATION
}

\author{
Ali Jamali ${ }^{*}$ and Francesc Antón Castro ${ }^{2}$ \\ ${ }^{1}$ Universiti Teknologi Malaysia (UTM), Faculty of Geoinformation and Real Estate- ali.jamali.65@gmail.com, \\ ${ }^{2}$ Yachay Tech University, School of Mathematics and Information Technology- fcastro@yachaytech.edu.ec
}

KEYWORDS: Homotopy continuation, 3D Data Interpolation, DEM, Optimization, Mathematics, ASTER GDEM

\begin{abstract}
:
In Geographic Information Science, polynomial methods such as linear estimation and non-polynomial methods including Inverse Distance Weighting and Kriging have been used for elevation data interpolation. In this paper, 3D data interpolation using linear and non-linear homotopy continuation as well as advanced polynomial interpolation methods are researched. Continuous deformations that reconstruct straight lines or algebraic curves between any pair of 3D data are presented. The implemented topological mathematical algorithm for 3D elevation data interpolation is compared to Inverse Distance Weighting and Triangulated Irregular Network (TIN) methods. The presented linear and non-linear mathematical algorithms show better results compared to Inverse Distance Weighting and TIN in terms of Root Mean Square Error and L-infinity.
\end{abstract}

\section{INTRODUCTION}

Geographic Information Science (GIS) could be defined as an integration of hardware, software, data and humans to analyse, integrate and manage geographically referenced information. In this definition, humans are included because just having the hardware and software cannot produce the desired result unless it is managed by an expert. In a GIS environment, the user is able to view, recognize, infer, examine and control spatial data in many ways to show relationships, patterns and trends linking the examined spatial data (Jamali, 2012).

The 3D GIS modelling technology can generate a more functional and insightful perspective. In 3D GIS, surface reconstruction is one of the most important aspects. Surface reconstruction is an old issue (Sharma and Anton, 2011). Several researches including Keppel (1975) and Fushs et al. (1977) addressed the issue. Hoppe et al. (1992) addressed the issue of object reconstruction from point cloud data. For surface reconstruction, Carr et al. (2001) used radial basis function and Amenta and Bern (1999) used Voronoi diagrambased filtering.

The Digital Elevation Model (DEM) derived from the Shuttle Radar Topography Mission (SRTM) in February 2000 and its other versions are one of the most important free spatial datasets which contain void values (Reuter et al., 2007). Advanced Spaceborne Thermal Emission and Reflection Radiometer Global Digital Elevation Map (ASTER GDEM) is another free spatial dataset which was released in October 2011. Void values are required to be calculated with data interpolation algorithms. For small and medium sized void areas, Kriging (Stein, 2012) and Inverse Distance Weighting (IDW) (Lu et al., 2008) are the most appropriate choices. In flat areas, spline interpolation for small and medium sized areas with high altitude is the proper algorithm. In flat areas with large void values, the TIN and IDW are the proper choices. For large void values in other terrains, an advanced spline method is required (Reuter et al., 2007).

Topological surface reconstruction from elevation data has not been addressed properly. Topological and geometrical surface reconstruction using continuous deformation (homotopy continuation) is introduced and discussed in this paper. This research intends to investigate $3 \mathrm{D}$ elevation data interpolation based on several advanced mathematics including linear nonlinear homotopy continuation.

Following this introduction, in Section 2, a literature review related to DEM and interpolation algorithms is presented. The study area and elevation data set are explained in Section 3. In Section 4, continuous deformation methods are discussed. Results of data interpolations are presented in Section 5. Section 6 presents conclusions and future research.

\section{BACKGROUND}

Laser scanning technology began in the 1990s (Amato et al., 2003) and it can measure a 3D object surface with a fast pulse. This technology is considered as a means of remote and quick data collection and it can be utilized in a wide range of applications from urban and regional planning (Sampson et al., 
2012; De Graff et al., 2012) to architecture (Lefsky and MacHale, 2008; Liu et al., 2012) as well as topography (Huising and Pereira, 1998; Chen et al., 2014), smart cities (Garnett and Adams, 2018), remote sensing (Lefsky et al., 2002) and automatic feature recognition (Xing et al., 2018).

Laser scanning includes three types of data acquisition: terrestrial laser scanning (TLS), mobile laser scanning (MLS) and aerial laser scanning (ALS). This technology (ALS/LiDAR) emits and captures signals returned from the surface of the Earth. An inertial measurement unit (IMU), a GNSS (GPS, GLONASS, COMPASS, or BEIDOU) unit and a laser scanning system are the three main parts of an ALS system (Tse et al., 2008). DEMs and Digital Surface Models (DSMs) can be captured by using Airborne Laser Scanning (ALS).

In spatial modelling and monitoring, DEMs are one of the most important sources of information (Reuter et al., 2007). Prediction of soil properties in environment and earth science, road construction in civil engineering and scene simulation in computer games are examples of DEM applicability (Hengl and Evans 2007).

Most of DEM data are required to be pre-processed to fill void values. Void values in elevation data have a bimodal distribution with peaks of the distribution, mostly in deep sloping areas and in flat areas (Gamache, 2004; Falorni et al., 2005).

DEM errors were categorized into three groups including gross error such as system malfunctions, systematic errors and random errors (Fisher and Tate, 2006). Void values are part of systematic errors which can be solved by interpolation algorithms.

Void filling methods are categorized into surface, volumetric (Vedera et al., 2003) and example-based (Sharf et al. 2004) algorithms. Linear estimation and cubic spline are examples of polynomial algorithms. Non-polynomial algorithms include Kriging and IDW. Hoffer et al. (2006) used an advanced cubic spline and compared its result graphically without a statistical comparison.

\section{STUDY AREA AND ELEVATION DATA SET}

The elevation data is from the Advanced Spaceborne Thermal Emission and Reflection Radiometer Global Digital Elevation Map Version 2 (ASTER GDEM 2) data set with longitude of 103.63402:103.64153 and latitude of 1.55597:1.56345 in the WGS84 coordinate reference system of Universiti Teknologi Malaysia (UTM) region (see Figure 1).

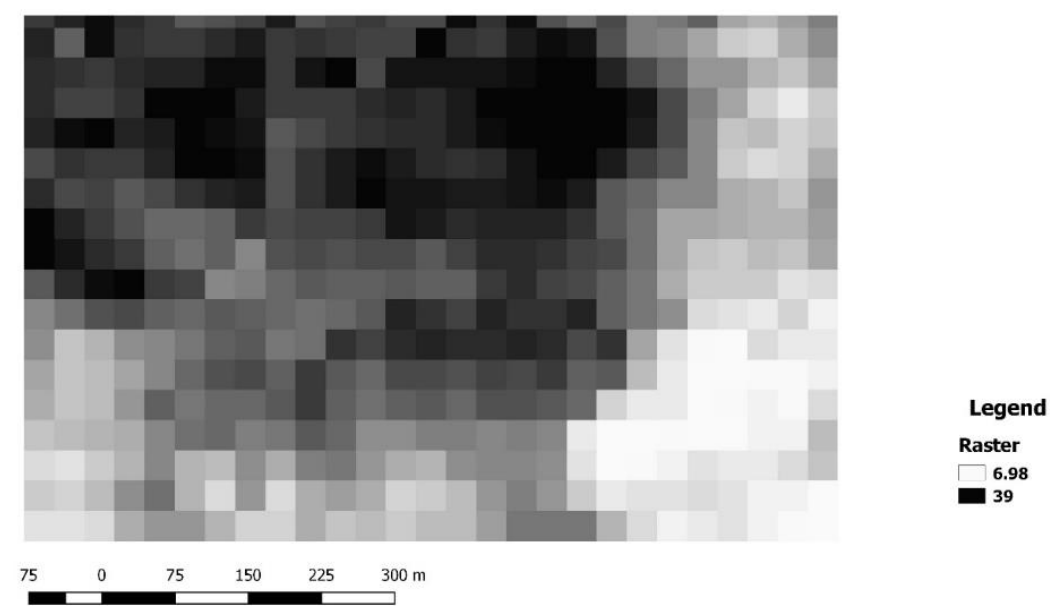

Figure 1. Elevation data extracted from SRTM data set.

\section{CONTINUOUS DEFORMATION}

Cubic interpolation, shape-preserving piecewise cubic interpolation and linear interpolation are three continuous interpolation algorithms. Cubic interpolation and shapepreserving piecewise cubic interpolation are in $\mathrm{C}^{2}$ and require at least four points for interpolation. Linear interpolation is in $\mathrm{C}^{0}$ and requires at least two points.

3D terrain modelling using piecewise cubic interpolation and smoothing piecewise cubic interpolation is shown in Figures 2 and 3 respectively. 


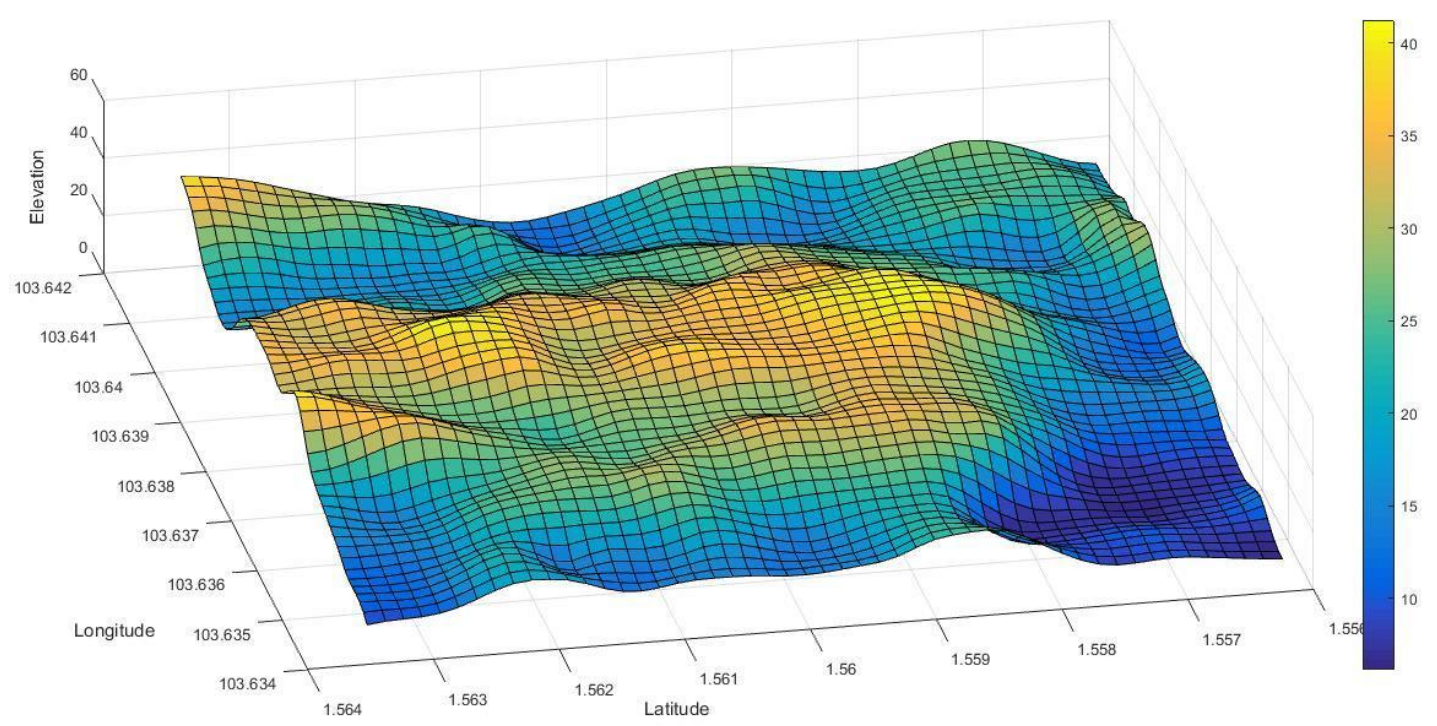

Figure 2. 3D terrain modelling from elevation data using piecewise cubic spline interpolation.

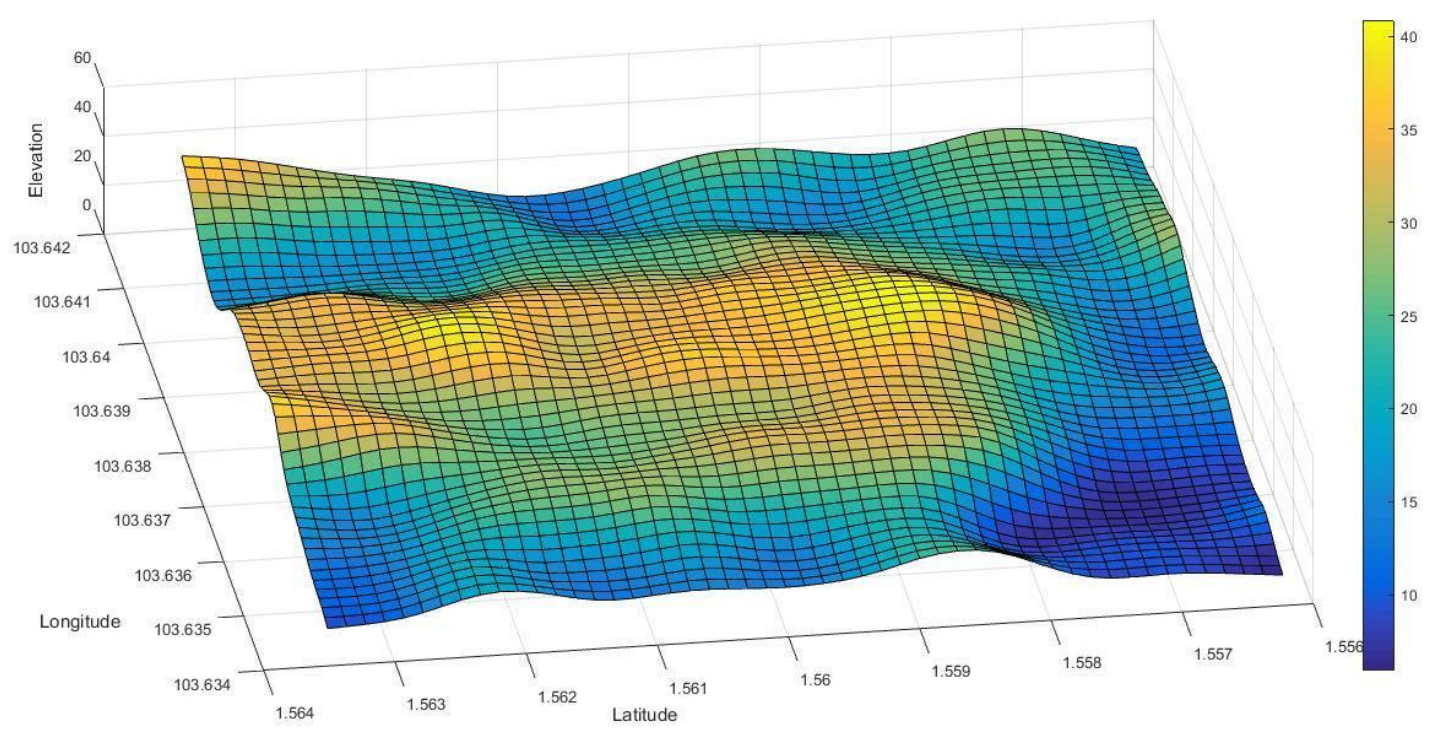

Figure 3. 3D terrain modelling from elevation data using smoothing piecewise cubic spline interpolation.

A homotopy is a continuous deformation of geometric figures or paths or more generally, functions: a function (or a path, or a geometric figure) is continuously deformed into another one (Allgower and Georg, 1990; Jamali et al., 2017; Jamali et al., 2018). A homotopy between two continuous functions $f_{0}$ and $\mathrm{f}_{1}$ from a topological space $\mathrm{X}$ to a topological space $\mathrm{Y}$ is defined as a continuous map $\mathrm{H}: \mathrm{X} \times[0,1] \rightarrow \mathrm{Y}$ from the Cartesian product of the topological space $\mathrm{X}$ with the unit interval $[0,1]$ to $Y$ such that $H(x, 0)=f_{0}$, and $H(x, 1)=f_{1}$, where $x \in X$. The two functions $f_{0}$ and $f_{1}$ are called, respectively, the initial and terminal maps. The second parameter of $\mathrm{H}, \lambda$, also called the homotopy parameter, allows for a continuous deformation of $\mathrm{f}_{0}$ to $\mathrm{f}_{1}$ (Allgower and Georg, 1990). Two continuous functions $f_{0}$ and $f 1$ are said to be homotopic, denoted by $f_{0} \simeq f_{1}$, if and only if there is a homotopy $\mathrm{H}$ taking $\mathrm{f}_{0}$ to $\mathrm{f}_{1}$. Being homotopic is an equivalence relation on the set $\mathrm{C}(\mathrm{X}, \mathrm{Y})$ of all continuous functions from $\mathrm{X}$ to $\mathrm{Y}$.

An equation of a linear homotopy is equal to (see Equation 1):

$H(x, \lambda)=(1-\lambda)$ f0 $(x)+\lambda f 1(x)$ where $\lambda \in[0,1]$

For a better understanding of piecewise cubic homotopy, an example is discussed as follows:

For a set of datasets with $\mathrm{f} 0=[0,0.13,0.14,0.50,0.90,1]$ and $\mathrm{f} 1=[0,0.15,0.13,0.60,0.94,1]$, the solutions of piecewise 
cubic and linear homotopies are equal to (see Figure 4,

Equations 2 and 3):

$\left(2.6434 x-88.1416 x^{3}\right)+\left(1690.9359 x^{3}-693.8402 x^{2}+92.8426 x-3.9086\right)+\left(-19.8757 x^{3}+24.7006 x^{2}-7.7530 x+0.7858\right)+$

$\left(5.3401 \mathrm{x}^{3}-13.1232 \mathrm{x}^{2}+11.158 \mathrm{x}-2.3661\right)+\left(-4.3173 \mathrm{x}^{3}+12.9520 \mathrm{x}^{2}-12.3088 \mathrm{x}+4.6741\right)$

$(1.15384 x)+(0.41-1.999 x)+(1.3055 x-0.0527)+(0.85 x+0.175)+(0.6 x+0.4)$

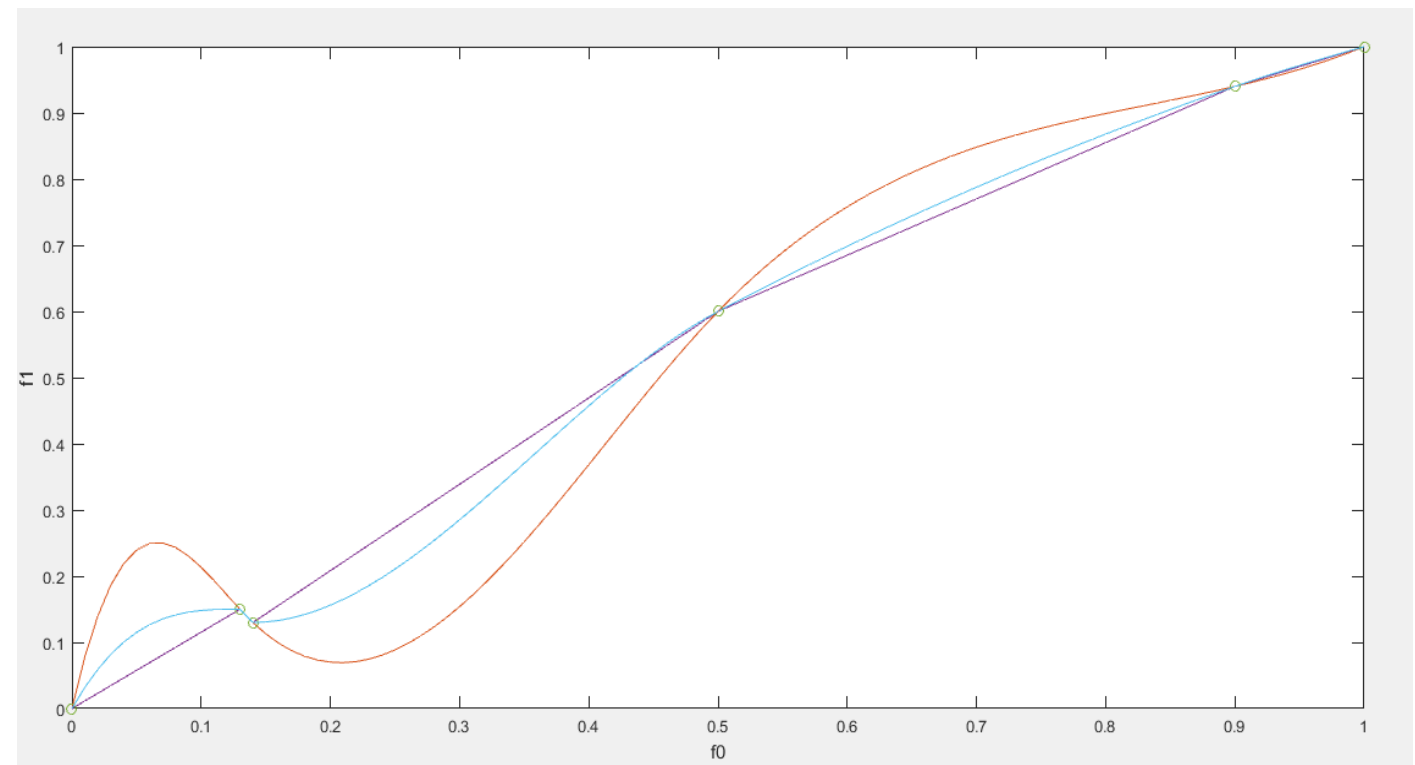

Figure 4. Piecewise cubic homotopy (orange color), Shape-preserving piecewise cubic homotopy (blue color) and linear homotopy (purple color) for a set of data.

As shown in Equation 2, Equation 3 and Figure 4, the solutions of homotopies for the example dataset contain five piecewise cubic and linear functions in five intervals between 0 and 1 from $\mathrm{f} 0$ to $\mathrm{f} 1$. For example, any interpolation value in the interval $[0.14 ; 0.50]$ is calculated by the $-19.8757 \mathrm{x}^{3}+24.7006$ $\mathrm{x}^{2}-7.7530 \mathrm{x}+0.7858$ cubic homotopy and the $1.3055 \mathrm{x}-$ 0.0527 linear homotopy.

\section{RESULTS}

In this research, 4208 points are used for elevation data interpolation and 468 points for the evaluation of the accuracy of the interpolations. Results of elevation data interpolation based on piecewise linear and cubic homotopy continuations are presented in Figures 5 and 6 respectively.
As can be seen in Figure 5, all elevation data belong to the [0; 1] interval.

Each elevation is calculated as follows (see Equation 4):

$E_{i}=\frac{E_{i}-E_{\min }}{E_{\max }-E_{\min }}$

Where Ei is the elevation at point $\mathrm{i}$ and $\mathrm{E}_{\min }$ is minimum elevation of the dataset and $E_{\max }$ is maximum elevation of the dataset. 


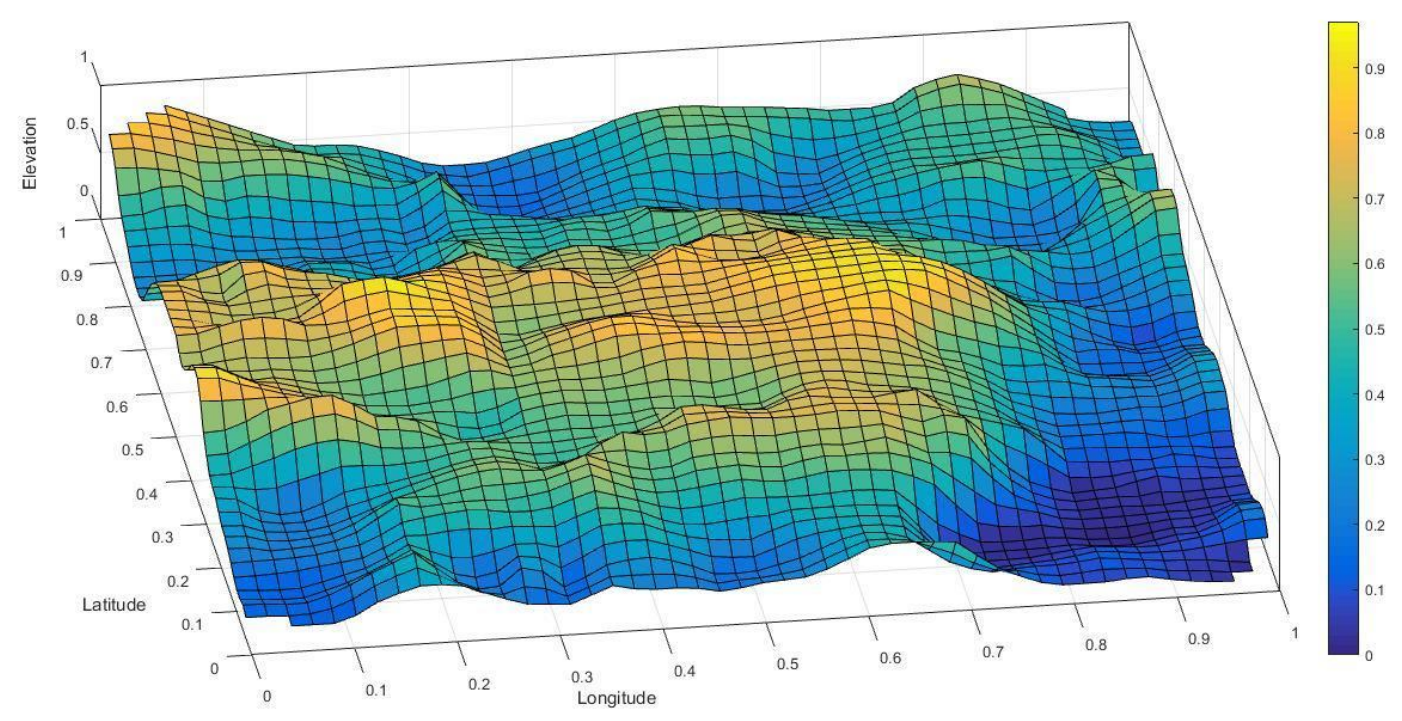

Figure 5. 3D terrain modelling from elevation data using homotopy continuation based on piecewise linear spline interpolation.

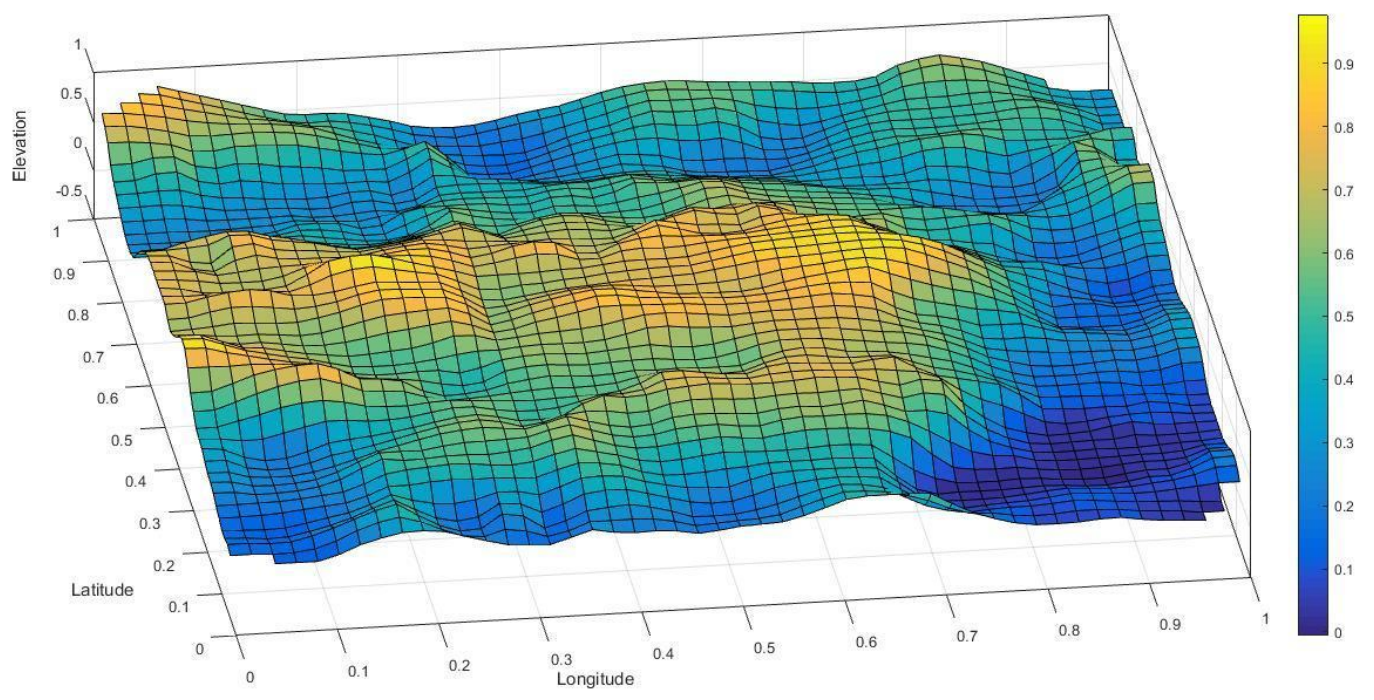

Figure 6. 3D terrain modelling from elevation data using homotopy continuation based on piecewise cubic spline interpolation.

Accuracy of interpolations using polynomial and nonpolynomial methods are based on Root Mean Square Error (RMSE) and L-infinity distance as follows (see Equation 5):

$R M S E=\sqrt{\frac{\sum_{i=1}^{n}\left(E_{\text {obs }, i}-E_{\text {interpolated }, i}\right)^{2}}{n}}$

Where $E_{o b s}$ is the real elevation data and $E_{\text {interpolated }}$ is the interpolated elevation from the IDW, TIN and continuous deformation.

L-infinity distance between two vectors or points A and B can be calculated (see Equation 6 and 7):

$L-\operatorname{infinity}(A, B)=\operatorname{MAX} X_{i}\left(\left|A_{i}-B_{i}\right|\right)$ $\lim _{k \rightarrow \infty}\left(\sum_{i=1}^{n}\left|A_{i}-B_{i}\right|^{k}\right)^{\frac{1}{k}}$

The results show an RMSE of 0.1984 meters for the cubic homotopy continuation and an RMSE of 0.2114 meters for linear the homotopy continuation. Two well-known nonpolynomial data interpolations including IDW and TIN are deployed to evaluate the results of the presented topological mathematical method in terms of RMSE.

The IDW method has an RMSE of 1.5439 meter for elevation data interpolation and the TIN method shows an RMSE of 7.5641 meters. Homotopy have L-infinity distance values of $1.32 \mathrm{~m}$ for cubic interpolation and $1.49 \mathrm{~m}$ for linear interpolation. IDW method has a value of $4.97 \mathrm{~m}$ and TIN shows an L-infinity distance value of $18.036 \mathrm{~m}$ (see Table 1). 


\begin{tabular}{|c|c|c|c|c|}
\hline $\begin{array}{c}\text { Interpolation } \\
\text { methods }\end{array}$ & $\begin{array}{c}\text { Linear } \\
\text { homotopy }\end{array}$ & $\begin{array}{c}\text { Cubic } \\
\text { Homotopy }\end{array}$ & IDW & TIN \\
\hline RMSE & 0.2114 & 0.1984 & 1.5439 & 7.5641 \\
L-infinity & 1.49 & 1.32 & 4.97 & 18.036 \\
\hline
\end{tabular}

Table 1. Accuracy of interpolation methods in term of RMSE and L-infinity

\section{CONCLUSIONS}

In this paper, 3D data reconstructions using continuous deformations for elevation data interpolation are presented and discussed. In previous GIS-based researches, topological interpolation algorithms were not researched properly, with IDW and TIN being the two popular interpolation algorithms.

Linear and cubic homotopies as advanced polynomial interpolation methods were introduced and compared to IDW and TIN. In the experiment, advanced mathematics shows an advantage over the conventional methods in terms of RMSE and L-infinity.

\section{REFERENCES}

Allgower, E. L., \& K. Georg, (1990). Numerical continuation methods: an introduction. Springer-Verlag New York, Inc. New York, NY, USA.

Amenta, N., Bern, M. (1999). Surface reconstruction byVoronoi filtering. Discrete Comput. Geom. 22(4), 481-504 (1999)

Carr, J.C., Beatson, R.K., Cherrie, J.B., Mitchell, T.J., Fright, W.R., McCallum, B .C. Evans,T.R.(2001). Reconstruction and representation of $3 \mathrm{D}$ objects with radial basis functions. In: Proceedings of the 28th Annual Conference on Computer Graphics and Interactive Techniques, pages, p. 76. ACM, New York.

Chen, X. T., Disney, M. I., Lewis, P., Armston, J., Han, J. T., $\&$ Li, J. C. (2014). Sensitivity of direct canopy gap fraction retrieval from airborne waveform lidar to topography and survey characteristics. Remote sensing of environment, 143, $15-25$.

De Graff, J. V., Romesburg, H. C., Ahmad, R., \& McCalpin, J. P. (2012). Producing landslide-susceptibility maps for regional planning in data-scarce regions. Natural hazards, 64(1), 729-749.

Falorni, G., Teles, V., Vivoni, E. R., Bras, R. L., \& Amaratunga, K. S. (2005). Analysis and characterization of the vertical accuracy of digital elevation models from the Shuttle Radar Topography Mission. Journal of Geophysical Research: Earth Surface, 110(F2).
Fisher, P. F., \& Tate, N. J. (2006). Causes and consequences of error in digital elevation models. Progress in physical Geography, 30(4), 467-489.

Fuchs, H., Kedem, Z.M., Uselton, S.P. (1977). Optimal surface reconstruction from planar contours. Commun. ACM 20(10), 693-702.

Gamache, M. (2004, September). Free and low cost datasets for international mountain cartography. In 4th ICA Mountain Cartography Workshop (Vol. 26).

Garnett' R., and Adams, M, D. (2018). LiDAR-A Technology to Assist with Smart Cities and Climate Change Resilience: A Case Study in an Urban Metropolis. ISPRS Int. J. GeoInf. 2018, 7(5), 161. https://doi.org/10.3390/ijgi7050161.

Gevrey, M., Dimopoulos, I., \&Lek, S. (2003). Review and comparison of methods to study the contribution of variables in artificial neural network models.Ecological modelling, 160(3), 249-264

Hengl, T. and Evans, I. S., (2007), Geomorphometry: A brief guide. In 9 Geomorphometry: Concepts, software, applications, T. Hengl and H. I. Reuter 10 (Eds.) (Ispra, Italy: European Commission, 2007), pp. 3-18.

Hofer, M., Sapiro, G., \& Wallner, J. (2006). Fair polyline networks for constrained smoothing of digital terrain elevation data. IEEE transactions on geoscience and remote sensing, 44(10), 2983-2990.

Huising, E. J., \& Pereira, L. G. (1998). Errors and accuracy estimates of laser data acquired by various laser scanning systems for topographic applications. ISPRS Journal of photogrammetry and remote sensing, 53(5), 245-261.

Ali Jamali, Francesc Antón Castro \& Darka Mioc (2018). A novel method of combined interval analysis and homotopy continuation in indoor building reconstruction, Engineering Optimization, DOI: 10.1080/0305215X.2018.1472253

Jamali, A., Anton, F., Abdul Rahman, A., and Mioc, D (2017). A COMPARISON OF ARTIFICIAL NEURAL NETWORK AND HOMOTOPY CONTINUATION IN 3D INTERIOR BUILDING MODELLING, Int. Arch. Photogramm. Remote Sens. Spatial Inf. Sci., XLII-4/W7, 13-21, https://doi.org/10.5194/isprs-archives-XLII-4-W7-13-2017.

Jamali, A. (2012). Semantic modelling for geo-database using CITYGML4J. Master thesis. Department of Geoinformation, Universiti Teknologi Malaysia, Malaysia.

Keppel, E. (1975). Approximating complex surfaces by triangulation of contour lines. IBM J. Res. Dev. 19(1), 2-11.

Lu, G. Y., \& Wong, D. W. (2008). An adaptive inversedistance weighting spatial interpolation technique. Computers \& geosciences, 34(9), 1044-1055. 
Liu, C., Li, W., Lei, W., Liu, L., \& Wu, H. (2011, October). Architecture planning and geo-disasters assessment mapping of landslide by using airborne lidar data and UAV images. In International Symposium on Lidar and Radar Mapping 2011: Technologies and Applications (Vol. 8286, p. 82861Q). International Society for Optics and Photonics.

Lefsky, M. A., Cohen, W. B., Parker, G. G., \& Harding, D. J. (2002). Lidar remote sensing for ecosystem studies: Lidar, an emerging remote sensing technology that directly measures the three-dimensional distribution of plant canopies, can accurately estimate vegetation structural attributes and should be of particular interest to forest, landscape, and global ecologists. AIBS Bulletin, 52(1), 19-30.

Lefsky, M. A., \& McHale, M. R. (2008). Volume estimates of trees with complex architecture from terrestrial laser scanning. Journal of Applied Remote Sensing, 2(1), 023521.

Ramon Moore, R. K. E., \& Cloud, M. J. (2009). Introduction to interval analysis. SIAM (Society for Industrial and Applied Mathematics), Philadelphia.

Reuter, H. I., Nelson, A., \& Jarvis, A. (2007). An evaluation of void-filling interpolation methods for SRTM data. International Journal of Geographical Information Science, 21(9), 983-1008.

Rudin, Walter (1980), Real and Complex Analysis (2nd ed.), New Delhi: Tata McGraw-Hill, ISBN 9780070542341.

Sampson, C. C., Fewtrell, T. J., Duncan, A., Shaad, K., Horritt, M. S., \& Bates, P. D. (2012). Use of terrestrial laser scanning data to drive decimetric resolution urban inundation models. Advances in water resources, 41, 1-17.

Sharf, A., Alexa, M., \& Cohen-Or, D. (2004, August). Context-based surface completion. In ACM Transactions on Graphics (TOG) (Vol. 23, No. 3, pp. 878-887). ACM.

Stein, M. L. (2012). Interpolation of spatial data: some theory for kriging. Springer Science \& Business Media.

Tse, R., Gold, C., \& Kidner, D. (2008). 3D City Modelling from LIDAR Data. Advances in 3D Geoinformation Systems, $161-175$.

Verdera, J., Caselles, V., Bertalmio, M., \& Sapiro, G. (2003, September). Inpainting surface holes. In Image Processing, 2003. ICIP 2003. Proceedings. 2003 International Conference on (Vol. 2, pp. II-903). IEEE.

Xing, X., Mostafavi, M, A. \& Chavoshi, s,H. (2018). A Knowledge Base for Automatic Feature Recognition from Point Clouds in an Urban Scene. ISPRS Int. J. GeoInf. 2018, 7(1), 28. https://doi.org/10.3390/ijgi7010028. 